\title{
BRONQUIOLITE OBLITERANTE E PNEUMONIA EM ORGANIZAÇÃO SECUNDÁRIA AO USO DE AMIODARONA
}

\section{BRONCHIOLITIS OBLITERANS ORGANIZING PNEUMONIA SECONDARY TO AMIODARONE}

DOI: $10.5380 /$ rmu.v2i3.43481.g26401

Gibran Avelino Frandoloso ${ }^{1}$, João Pedro Pereira da Cunha ${ }^{1}$, Caio Cesar Cervi Lagana ${ }^{1}$, Melissa Pupo ${ }^{1}$

\section{RESUMO}

A amiodarona é um antiarrítmico comumente utilizado na prática clínica e pode estar associado a diversos efeitos adversos, muitos deles com significativa morbimortalidade, como o acometimento pulmonar. O presente caso descreve uma mulher de 63 anos com toxicidade pulmonar aguda pela amiodarona, utilizada em curto período de tempo. A realização de biópsia pulmonar demonstrou bronquiolite obliterante e pneumonia em organização (BOOP). Após interrupção do uso do antiarrítmico e o início da corticoterapia, a paciente obteve expressiva melhora clínica e radiológica.

Palavras-Chave: Amiodarona, Pneumopatias, Toxicidade de drogas.

\section{ABSTRACT}

Amiodarone is a widely used anti-arrhythmic agent in clinical practice. One of the potentially life threatening adverse effects is amiodarone-induced pulmonary toxicity (APT). We describe a case of a 63-year-old woman with APT. The diagnostic workup included pulmonary biopsy that demonstrated bronchiolitis obliterans organizing pneumonia (BOOP). Amiodarone was discontinued subsequently and corticosteroid treatment initiated, with clinical and radiological disease remission.

Key-Words: Amiodarone, Lung Diseases, Drug toxicity. 


\section{INTRODUÇÃO}

A amiodarona é um antiarrítmico derivado do benzofurano com comprovada eficácia no tratamento de taquiarritmias ${ }^{1,2}$. Dentre o perfil de efeitos adversos com o uso dessa medicação destacam-se o comprometimento neuromuscular, hepático, tireoidiano, cutâneo, ocular (corneano) e pulmonar ${ }^{1,2,3}$. A toxicidade pulmonar induzida por amiodarona acomete cerca de $5 \%$ dos pacientes tratados, sendo o espectro de lesão bastante variado ${ }^{1,2,3}$. O presente relato de caso ilustra o raro acometimento pulmonar agudo induzido por amiodarona em padrão de bronquiolite obliterante com pneumonia em organização (BOOP).

\section{RELATO DO CASO}

Mulher de 63 anos foi admitida no serviço de Cirurgia Geral do HC-UFPR com plano de tratamento cirúrgico após investigação por queixa de dor abdominal tipo cólica em hipocôndrio direito, náuseas, vômitos, icterícia, acolia e colúria, que resultou em um diagnóstico de colangiocarcinoma em papila duodenal.

Previamente hígida, com histórico de anemia megaloblástica por deficiência de vitamina B12, adequadamente tratada e sem posterior investigação, com carga tabágica de 25 maços-ano e interrupção do tabagismo há 20 anos. Foi submetida à duodenopancreatectomia sem intercorrências e encaminhada à UTI no pós-operatório. No 2ํ dia pósoperatório, evoluiu com fibrilação atrial de alta resposta, sendo tratada com $900 \mathrm{mg}$ de Amiodarona via endovenosa em 24 horas, com posterior reversão para ritmo sinusal. No 5o dia de pós-operatório foi submetida a uma laparotomia exploradora para drenagem de coleção em fundo gástrico, com boa resposta. No 14ㅇ dia pós-operatório, evoluiu com dispneia e tosse seca. Ao exame físico, apresentava-se em regular estado geral, lúcida e orientada em tempo e espaço, Glasgow 15, hipocorada +/+4, hidratada, temperatura $36.4^{\circ} \mathrm{C}$, frequência respiratória de 24 incursões por minuto, saturação de oxigênio de $92 \%$ com névoa à $4 \mathrm{l} / \mathrm{min}$. Ausculta pulmonar com murmúrio vesicular presente bilateralmente e presença de estertores finos difusos, mais proeminentes em região de terço superior de hemitórax à direita. Restante do exame sem particularidades. Foi iniciada antibioticoterapia com Meropenem e Vancomicina. Raio-X de tórax demonstrou infiltrado em lobo superior direito. A paciente não apresentou febre e o hemograma não apresentou leucocitose/leucopenia ou desvio à esquerda. Realizada tomografia de tórax que demonstrou achados compatíveis com BOOP (bronquiolite obliterante e pneumonia em organização) (figura 1). A TC de tórax realizada no préoperatório para estadiamento da neoplasia encontrava-se normal (figura 2).

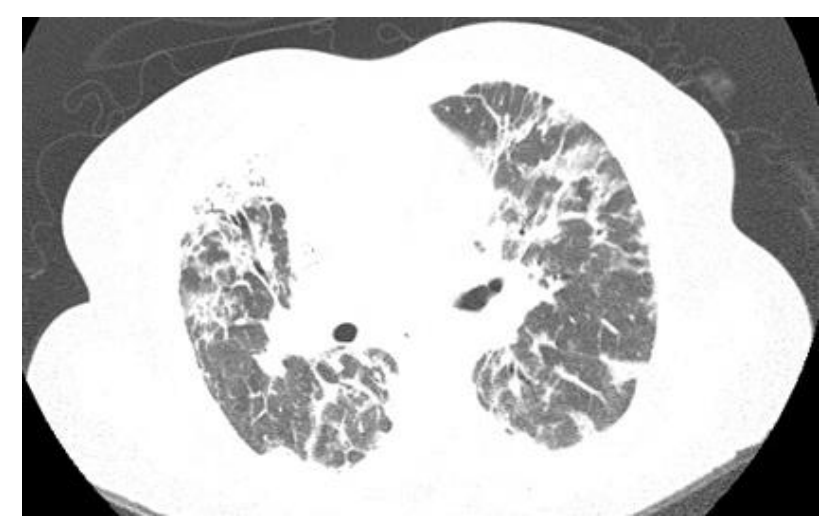

FIGURA 1. Tomografia computadorizada de tórax com presença de infiltrado reticular subpleural associado com áreas de atenuação em vidro fosco bilateralmente, sugerindo doença pulmonar intersticial.

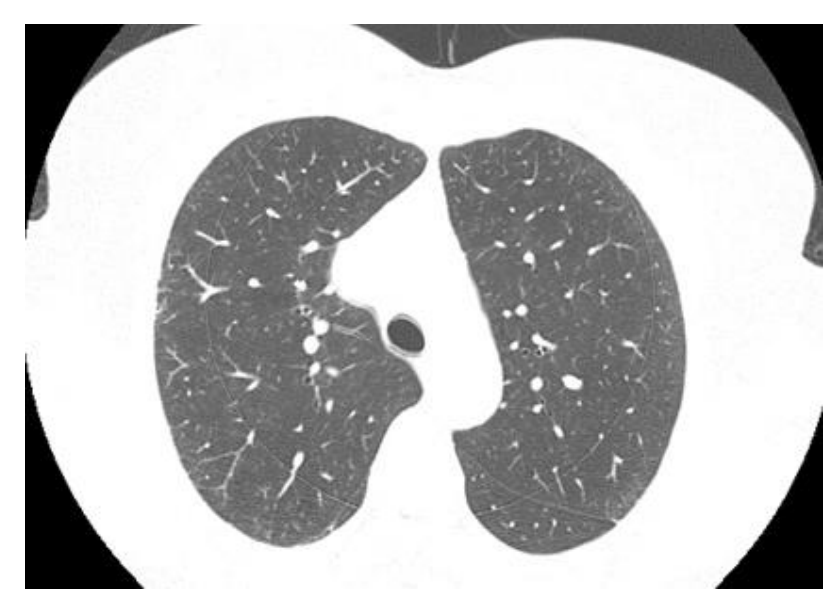

FIGURA 2. Tomografia de tórax sem alterações

Sem resposta após 4 dias de antibioticoterapia, micafungina foi adicionada ao esquema terapêutico e foi solicitado broncofibroscopia com lavado broncoalveolar (tabela 1) e biópsia. Aventada a hipótese de BOOP por amiodarona, foi iniciado metilprednisolona $62,5 \mathrm{mg}$ via endovenosa de 
12 em 12 horas. Após 3 dias do início da corticoterapia, a paciente evoluiu com melhora dos sintomas respiratórios, com redução da necessidade de oxigenoterapia para $21 / \mathrm{min}$, saturação de oxigênio de $97 \%$ e frequência respiratória de 16 . O corticoide foi então reduzido para $62,5 \mathrm{mg}$ no sétimo dia de tratamento e a paciente não necessitou mais de oxigênio suplementar.

\begin{tabular}{ll}
\hline CELULARIDADE & RESULTADO \\
\hline Células nucleadas & $50 / \mathrm{uL}$ \\
Leucócitos & $22.5 / \mathrm{uL}$ \\
Neutrófilos & $8 \%$ \\
Linfócitos & $37 \%$ \\
Células metaplásicas & $18 \%$ \\
Eosinófilos & $18 \%$ \\
Macrófagos & $19 \%$ \\
\hline
\end{tabular}

TABELA 1. Lavado Broncoalveolar com resultado de celularidade

Amostras foram biopsiadas e enviadas para o setor de patologia. As lesões pulmonares foram reavaliadas com nova TC de tórax, em que se verificou significativa regressão dos achados constatados previamente. O gram, cultura para germes comuns, micológico direto, cultura para fungos, cultura para anaeróbios, bacterioscopia por Ziehl-Neelsen foram negativos tanto no LBA quanto na biópsia e as hemoculturas não positivaram. Os antibióticos foram suspensos, o corticoide foi substituído por prednisona $60 \mathrm{mg} /$ dia via oral.

A biópsia pulmonar via broncofibroscopia demonstrou parede de vias aéreas com inflamação e edema discretos, tecido pulmonar com BOOP (bronquiolite obliterante e pneumonia em organização), fibrose intersticial discreta a moderada, hiperplasia de pneumócitos, macrófagos intraalveolares em pequena quantidade e focos de antracnose. Os achados, apesar de inespecíficos, já foram descritos em casos de pneumonite por amiodarona. No 47으 dia de pós-operatório, a paciente foi de alta em uso de prednisona $40 \mathrm{mg} / \mathrm{d}$ com seguimento no ambulatório da Pneumologia, assintomática do ponto de vista respiratório.

\section{DISCUSSÃO}

A amiodarona apresenta entre seus efeitos adversos a toxicidade pulmonar, principalmente associada ao uso crônico e a altas doses do medicamento, sendo os maiores fatores de risco o uso de $400 \mathrm{mg} /$ dia de amiodarona por mais de 2 meses ou $200 \mathrm{mg} /$ dia por $2 \operatorname{anos}^{4,5}$. Outros fatores de risco incluem gênero masculino, doença pulmonar préexistente, idade avançada, exposição a altas concentrações de oxigênio ${ }^{1,2,5,6,8}$. Pneumonite aguda associada ao uso de amiodarona por curto período de tempo é muito mais rara. Apesar de existirem relatos de casos na literatura de toxicidade pulmonar associada ao uso de amiodarona por poucos dias, ou mesmo horas, essa é uma situação rara cujo diagnóstico em geral não é realizado prontamente, o que pode retardar o tratamento ${ }^{2,3,4}$. Além de pouco frequente, a pneumonite por amiodarona tem como diagnósticos diferenciais uma série de condições com sintomatologia muito parecida, como pneumonia infecciosa, falência cardíaca, tromboembolismo pulmonar, doença pulmonar restritiva, edema pulmonar, o que torna seu diagnóstico ainda mais difícil $^{2,9}$.

Como não há marcador para essa doença, e os sinais e sintomas são inespecíficos, sem a biópsia pulmonar o diagnóstico acaba sendo de exclusão ${ }^{5,7}$. Entre os sintomas estão dispneia, tosse seca, dor torácica pleurítica. A tomografia computadorizada de tórax geralmente apresenta acometimento pulmonar bilateral'.

A gravidade da doença, com altas taxas de mortalidade ou sequela, como fibrose pulmonar, quando há retardo no início do tratamento apropriado justificam a importância do tema, apesar de ser uma condição rara ${ }^{6}$. Quando a síndrome do desconforto respiratório agudo ocorre, a mortalidade pode variar de 1 a $50 \%^{8}$.

O tratamento é baseado na suspensão imediata do uso de amiodarona e no uso de glicocorticoides por um período que varia de 2 a 6 meses ${ }^{4}$.

No relato de caso apresentado podemos evidenciar que o diagnóstico e tratamento adequados não foram realizados imediatamente após o aparecimento dos sintomas. A primeira hipótese foi de infecção pulmonar, o que justifica o tratamento inicial 
com meropenem e vancomicina. Só após a não melhora clínica da paciente mesmo em uso de antibioticoterapia de largo espectro é que se considerou a possibilidade de pneumonite por amiodarona. A ausência de febre, leucocitose ou leucopenia no hemograma ajudaram a descartar o diagnóstico de infecção pulmonar e buscar outra causa para o quadro clínico exposto.

Como a toxicidade pulmonar aguda por amiodarona é rara e não há marcadores específicos para a doença, sendo um diagnóstico de exclusão, justifica-se o uso empírico de antibioticoterapia. Uma vez observada a não melhora do quadro clínico, a hipótese de efeito adverso medicamentoso deve ser levantada e a terapêutica específica instituída. Alguns autores defendem que a primeira intervenção seja a terapia direcionada para os prováveis diagnósticos diferenciais, e só após a falha terapêutica a toxicidade pulmonar por amiodarona seja uma suspeita ${ }^{9}$. A melhor evidência do acerto do diagnóstico seria a evolução favorável da paciente após suspensão da amiodarona e início da corticoterapia.

Embora o uso crônico e em altas doses de amiodarona sejam os principais fatores de risco para a toxicidade pulmonar, é importante lembrar que os efeitos adversos desse medicamento podem ocorrer mesmo após um curto período de tempo de uso, como foi o caso exposto no presente relato de caso. 0 diagnóstico de BOOP após a apresentação clínica descrita, exames de TC de tórax e biópsia pulmonar ocorreu em uma mulher sem história de doença pulmonar prévia, que não fazia uso de oxigenioterapia e o uso de amiodarona foi por curto período de tempo. O tratamento instituído foi eficaz, com remissão do quadro clínico e melhora dos parâmetros radiológicos, cenário que corrobora a boa evolução da BOOP por amiodarona quando há interrupção do uso do antiarrítmico e início da corticoterapia o mais breve possível $^{9}$.

\section{CONCLUSÃO}

O caso relatado ilustra um raro padrão de lesão pulmonar intersticial associado ao uso de amiodarona. O diagnóstico de certeza foi possível somente pela realização de biópsia pulmonar. Vale ressaltar a importância da inclusão dentre os diagnósticos diferenciais do presente quadro clínico a clássica, porém incomum, toxicidade pulmonar por amiodarona, mesmo quando utilizada por breves períodos de tempo. O tratamento com interrupção do uso do antiarrítmico e início da corticoterapia foi eficaz para a favorável evolução clínica da paciente.

\section{REFERÊNCIAS}

1. Boriani G, Ferruzzi L, Corti B, Ruffato A, Gavelli G, Mattioli S. Short-term onset of fatal pulmonary toxicity in a patient treated with intravenous amiodarone for post-operative atrial fibrillation. International Journal of Cardiology . 2012; 159: 1-4

2. Lee $W$, et al. Very early onset of amiodaroneinduced pulmonary toxicity. Korean Circulation Journal. 2013; 43: 699-701.

3. Ferreira PG, Saraiva F, Carreira C. Toxicidade pulmonar aguda precoce associada à amiodarona. Acta Med Port. 2012; 25(5):342-345

4. Oh E, et al. Acute amiodarone-induced lung toxicity. American Journal of the Medical Sciences. 2015; 349: 89

5. Nacca N, Bhamidipati CM, Yuhico LS, Pinnamaneni $S$, Szombathy T. Severe amiodarone induced pulmonary toxicity. J Thorac Dis. 2012; 4(6):667-70.

6. Hudzik B, Polonski L. Amiodarone-induced pulmonary toxicity. Canadian Medical Association jornal. 2012, 184(15): 819

7. Camus $\mathrm{P}$, et al. Amiodarone pulmonary toxicity. Clin Chest Med. 2005; 25: 65-75 Mankikian J, et al. Initial characteristics and outcome of hospitalized patients with amiodaronepulmonary toxicity. Respiratory Medicine. 2014; 108: 638-646

8. Mankikian J, Favelle O, Guillon A. Initial characteristics and outcome of hospitalized patients with amiodarone pulmonary toxicity. Respiratory Medicine. 2014; 108: 638-646

9. Van Cott, et al. Amiodarone-induced pulmonary toxicity: Case study with syndrome analysis. Heart \& Lung. 2013; 42: 262-266 